\title{
Numerical analysis of steady-state and transient charge transport in organic semiconductor devices
}

\author{
Evelyne Knapp • Beat Ruhstaller
}

Received: 21 September 2010 / Accepted: 4 February 2011 / Published online: 18 February 2011

(C) Springer Science+Business Media, LLC. 2011

\begin{abstract}
A one-dimensional numerical model for the simulation of organic semiconductor devices such as organic light-emitting devices and solar cells is presented. The model accounts for the energetic disorder in organic semiconductors and assumes that charge transport takes place by a hopping process between uncorrelated sites. Therefore a Gaussian density of states and the use of the Fermi-Dirac statistics are introduced. The model includes density-, field- and temperature- dependent mobilities as well as the generalized Einstein relation. The numerical methods to solve the underlying drift-diffusion problem perform well in combination with the novel physical model ingredients. We demonstrate efficient numerical techniques that we employ to simulate common experimental characterization techniques such as current-voltage, dark-injection transient and electrical impedance measurements. This is crucial for physical model validation and for material parameter extraction. We also highlight how the numerical solution of the novel model differs from the analytical solution of the simplified drift-only model.
\end{abstract}

Keywords Numerical simulation · Organic light-emitting device · Small signal analysis · Extended Gaussian disorder model

\section{Introduction}

While organic LEDs have started to succeed commercially in displays and lighting, further progress in terms of efficiency and lifetime is still needed. One valuable approach is to elaborate and refine physical models and numerical methods. Theoretical models for describing

E. Knapp $(\varangle) \cdot$ B. Ruhstaller

Institute of Computational Physics, Zurich University of Applied Sciences, Wildbachstr. 21, 8401 Winterthur, Switzerland

e-mail: evelyne.knapp@zhaw.ch

B. Ruhstaller

Fluxim AG, Dorfstrasse 7, 8835 Feusisberg, Switzerland

e-mail: beat.ruhstaller@fluxim.com 
charge transport and recombination have been introduced (Scott and Malliaras 1999; Crone et al. 2000; Blom et al. 1996; Ruhstaller et al. 2001, 2003) several years ago. Electrical characterization of devices and materials is essential as it helps to elucidate the underlying, physical models of charge carrier transport in disordered, organic semiconductors. Besides the commonly used current-voltage curves (van Mensfoort et al. 2008), dark-injection measurements and impedance spectroscopy offer additional ways to validate models for organic LEDs and extract model parameters. By means of a one-dimensional numerical OLED model we are able to simulate these different operating conditions. In this paper, we present numerical methods in the physical and numerical framework of reference (Knapp et al. 2010) and solve directly for both the steady- and transient state. Further, we conduct a numerical small signal-analysis for OLEDs. The drift-diffusion equations are solved in a coupled manner. The disordered nature of organic semiconductors affects the density of states (DOS), the mobility model, the Einstein relation as well as charge injection. These novel physical model ingredients are integrated in the numerical solver.

\section{Transport model}

\subsection{Governing equations}

To simulate charge transport in organic semiconductor devices we use the continuum approach and solve the semiconductor drift-diffusion equations (Van Mensfoort and Coehoorn 2008). Since our analysis is restricted to hole transport, we can describe Poisson's (1) by the electric potential $\psi$ and the hole densities $p$. The elementary charge is denoted by $q$, the permittivity by $\epsilon$. To describe the conservation of charge carriers, we use the continuity (2)

$$
\begin{aligned}
& F_{\psi}(\psi, p)=\nabla \cdot(\epsilon \nabla \psi)-q(-p)=0, \\
& F_{p}(\psi, p)=-\nabla \cdot J_{p}=q \frac{\partial p}{\partial t} .
\end{aligned}
$$

The hole current in (3) can be split in a drift current due to the electric field and a diffusion current

$$
J_{p}=-q p \mu_{p} \nabla \psi-q D_{p} \nabla p .
$$

The mobility $\mu_{p}$ is field-, temperature- and density-dependent. The diffusion coefficient $D_{p}$ is also density-dependent and described by the generalized Einstein relation.

\subsection{Physical model of organic semiconductors}

As opposed to inorganic semiconductors transport in polymers and small molecules is assumed to occur via a hopping process between uncorrelated sites. Therefore the density of states of mobile charge carriers for organic semiconductors is represented by a Gaussian DOS

$$
N_{\text {Gauss }}(E)=\frac{N_{0}}{\sqrt{2 \pi \sigma^{2}}} \exp \left[-\left(\frac{E-E_{0}}{\sqrt{2} \sigma}\right)^{2}\right]
$$

with the site density $N_{0}$, the width of the Gaussian $\sigma$ and the reference energy level $E_{0}$. In contrast to inorganic semiconductors where in unconfined systems parabolic bands describe 
the DOS, organic semiconductor are more likely to become degenerate (Preezant et al. 2002). Thus the Fermi-Dirac statistics is applied instead of the Boltzmann approximation. The shape of the Gaussian DOS in (4) affects the mobility of charge carriers and the diffusion coefficient. Therefore a generalized Einstein relation must be considered (Roichman and Tessler 2002) for disordered organic materials. Van Mensfoort and Coehoorn (2008) introduced a density-dependent enhancement function $g_{3}(p, T)$ which is multiplied to the diffusion coefficient

$$
D(p, F, T)=\frac{k T}{q} \mu(p, F, T) \times g_{3}(p, T) .
$$

Especially at high carrier densities and high disorder parameter $\sigma$, the generalized Einstein relation differs significantly from the classical Einstein relation $D=\mu k T / q$.

Further, the mobility model consists of a field-, temperature- and density-dependent part. This model is called Extended Gaussian Disorder Model (EGDM) (Van Mensfoort and Coehoorn 2008; Pasveer et al. 2005) and can be written as a product of a mobility pre-factor $\mu_{0}(T)$ and the density-enhancement function $g_{1}(p, T)$ and the field-enhancement function $g_{2}(F, T)$ as given in (Van Mensfoort and Coehoorn 2008):

$$
\mu(p, F, T)=\mu_{0}(T) \times g_{1}(p, T) \times g_{2}(F, T)
$$

where $p$ denotes the density, $F$ the electric field and $T$ the temperature. The enhancement functions $g_{1}(p, T), g_{2}(F, T)$ and $g_{3}(p, T)$ are nonlinear and increase more strongly, the bigger the disorder in the organic material is. For the application of the EGDM three parameters are to be specified in case of single-carrier transport: the site density $N_{0}$, the width of the DOS $\sigma$, and the mobility pre-factor $\mu_{0}(T)$.

\section{Numerical method}

The drift-diffusion equations (1-2) with the organic model ingredients are discretized with the finite volume method, the current expression (3) with the Scharfetter-Gummel discretization (1969). The resulting system of discretized equations is then solved in a coupled manner with Newton's algorithm (Knapp et al. 2010) for the transient as well as the steady-state case. In the coupled algorithm (Newton) the components (1-2) are solved simultaneously for all degrees of freedom. For the steady-state the time-dependent right-hand side of (2) is set to zero. For the transient case the implicit Euler method is applied to solve the time-dependent equations. We assume Dirichlet boundary conditions for the density and the potential for all simulation examples presented here.

Regarding the small-signal analysis, the steady-state voltage $V_{0}$ is modulated with a sinusoidal voltage of amplitude $V^{a c}$ and with angular frequency $\omega: V=V_{0}+V^{a c} e^{i \omega t}$. The potential $\psi$ and the hole densities $p$ can be expanded into a steady-state and harmonic term under the assumption of the small-signal analysis

$$
\begin{aligned}
& \psi(x, t)=\psi_{0}(x)+\psi^{a c}(x) e^{i \omega t} \\
& p(x, t)=p_{0}(x)+p^{a c}(x) e^{i \omega t}
\end{aligned}
$$

where the ac components are complex-valued. To solve the following small-signal equations, the solution of the dc problem for $V=V_{0}$ needs first to be calculated. The above quantities (7-8) are then inserted into the semiconductor equations (1-2) which can be transformed into a linear system of equations for the unknown ac components $\psi^{a c}$ and $p^{a c}$. From the solution of the linear system the ac current density $J_{p}^{a c}$ can be determined. The displacement 
Fig. 1 Energy level diagram of the symmetric hole-only device: The HOMO energy level is aligned with the work functions of the anode and cathode and allows a good hole injection

Table 1 Simulation parameter set of the hole-only device for the three operating conditions

\begin{tabular}{lll} 
& \multicolumn{2}{c}{ LUMO } \\
& & \\
& & \\
& & \\
& & \\
& & \\
& & \\
\hline Parameter & Value & Cathode \\
\hline$N_{0}$ & $8.5 \times 10^{26}$ & Units \\
$\sigma$ & 0.15 & $\mathrm{~m}^{-3}$ \\
$\mu_{0}(T)$ & $10^{-10}$ & $\mathrm{eV}^{2}$ \\
$p(0)$ & $0.5 \times N_{0}$ & $\mathrm{~m}^{2} \mathrm{~V}^{-1} \mathrm{~s}^{-1}$ \\
$p(L)$ & $0.5 \times N_{0}$ & $\mathrm{~m}^{-3}$ \\
\hline & & $\mathrm{m}^{-3}$ \\
\hline
\end{tabular}

current $J_{D}^{a c}=\epsilon \frac{\partial E^{a c}}{\partial t}$ is added to the current component which results in a spatially constant total current density $J^{a c}$. From the complex admittance $Y=J^{a c} / V^{a c}$, the small-signal capacitance $C$ and conductance $G$ can be obtained. This method offers a fast and efficient calculation of the admittance as for each frequency only a linear system of equations for the ac components needs to be solved.

\section{Simulation results}

\subsection{Device structure and simulation parameters}

In the following a symmetric hole-only device as shown in Fig. 1 is simulated under three different operating conditions. The highest occupied molecular orbital (HOMO) of the device is aligned with the work functions of the anode and cathode. Therefore, we obtain a wellinjecting anode and cathode. The high barrier for injection of electrons from the cathode to the lowest unoccupied molecular orbital (LUMO) level essentially leads to a hole-only device.

In Table 1 we list the parameters used in the following simulations. The hole charge densities at the anode and cathode $(p(0)$ and $p(L))$ are set to half of the density of chargeable sites $N_{0}$, i.e. the Gaussian DOS is half-filled at either side due to the energy level alignment at the electrodes.

\subsection{Steady-state current-voltage curves}

In a first step, we calculate the steady-state for different voltages and obtain current-voltage characteristics as shown in Fig. 2. We compare the steady-state results for the EGDM and the constant mobility case with the analytical solution which is given by Mott and Gurney (1938)

$$
J(V)=\frac{9}{8} \varepsilon \varepsilon_{0} \mu_{0} \frac{V^{2}}{L^{3}}
$$

where $L$ denotes the length of the device, $V$ the applied voltage and $\mu_{0}$ the mobility. All of the analytical solutions in the following are obtained by neglecting the diffusion and only 


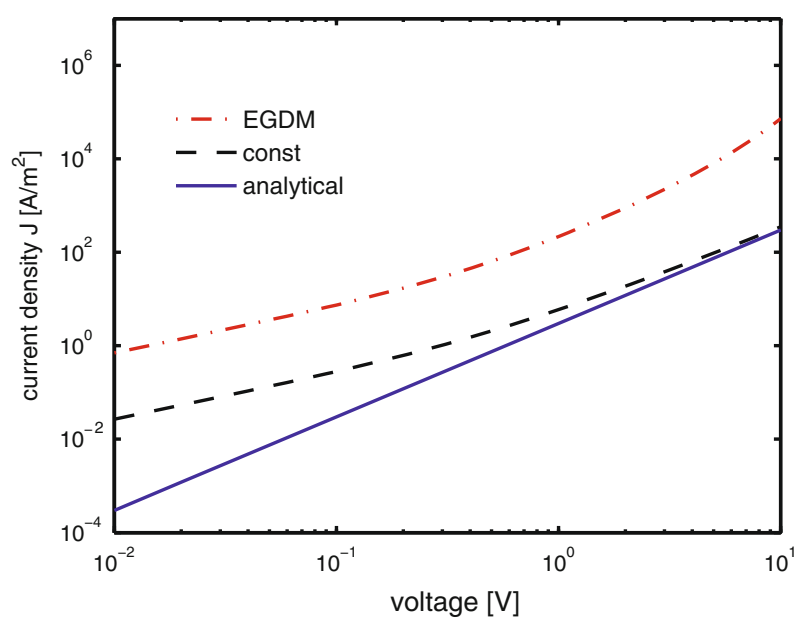

Fig. 2 Current-voltage curve for a hole-only device for a constant mobility and diffusion coefficient, the EGDM and the analytic solution (Mott-Gurney)

taking the drift in (3) into account. We observe the effect of diffusion especially at low voltages where the current is increased. In the regime of high bias the analytical solution and the constant mobility case merge whereas the EGDM solution moves away due to the field- and density-dependence of the EGDM and the slope of the EGDM curve is increased. Generally, the current density of the EGDM is higher due to the enhancement functions $g_{1}(p, T), g_{2}(F, T)$ and $g_{3}(p, T)$. The EGDM has recently been successfully applied to current-voltage curves by van Mensfoort et al. (2008). A comprehensive analysis of the effects of the EGDM and its components for the steady-state is conducted in reference (Van Mensfoort and Coehoorn 2008).

\subsection{Dark-injection transients}

We now turn to the analysis of the time-dependent response to a step voltage. We perform transient simulations for the same device as above shown in Fig. 3. The dark-injection transients are simulated from 2 to $12 \mathrm{~V}$ in steps of $2 \mathrm{~V}$. For the initial rise of the transient response the analytical solution is described by Helfrich and Mark (1962)

$$
J(t)=\frac{2 L \varepsilon \varepsilon_{0}}{\mu} \frac{1}{\left(2 \tau_{S C F}-t\right)^{2}}
$$

where the space-charge-free transit time is defined as $\tau_{S C F}=\frac{L^{2}}{\mu V}$. In the SCLC at the transit time $\tau_{t}=0.786 \tau_{S C F}$ the maximum current is obtained and reaches a value of $J\left(\tau_{t}\right)=$ $1.21 J_{c}$ where $J_{c}$ stands for the stationary current from Mott-Gurney's law (9). The value of $J\left(\tau_{t}\right)=1.21 J_{c}$ is marked in Fig. 3 with a star. Note that the slope of the line connecting these stars is minus two which can be explained with (9). The relatively low charge mobility of organic semiconductors leads to space-charge limited transport. In this regime the transient response exhibits a cusp that relates to the transit time and thus the charge mobility. This has been the motivation for carrying out dark-injection transient experiments.

We notice that the peak position for the constant mobility case coincides well with the analytical solution, especially at high bias where the drift current is the dominant part of the total current. At low voltage the agreement becomes worse due to the diffusion current. 


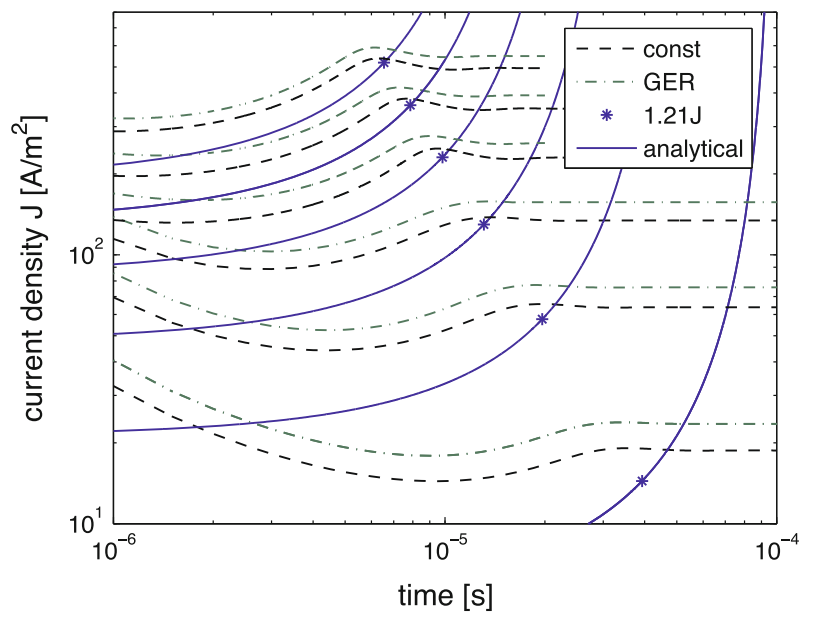

Fig. 3 Dark-injection transients for a hole-only device for a constant mobility and diffusion coefficient, the analytical solution and the generalized Einstein solution (GER). The transients were simulated from 2 to $12 \mathrm{~V}$

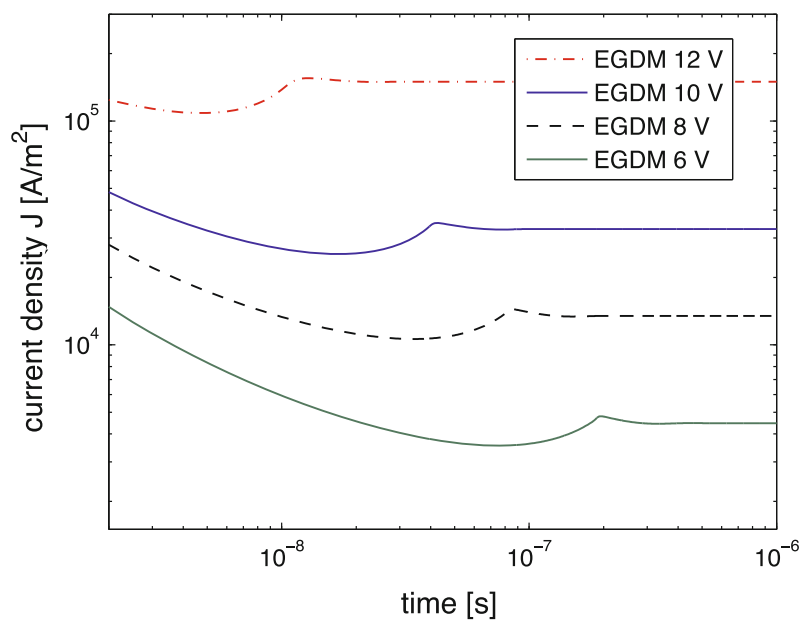

Fig. 4 Dark-injection transients for a hole-only device for the EGDM at different voltages

By introducing the generalized Einstein relation (GER) we see an increased current. However, the position of the peak is not remarkably shifted.

If we also consider the enhancement functions for the EGDM as shown in Fig. 4, the peak positions shift to shorter times due to the enhanced mobility and the current density is vastly increased. In the EGDM case no formulas are available that would relate the peak position in the current with EGDM mobility parameters. Therefore, we have to rely on numerical fitting of the model parameters. 


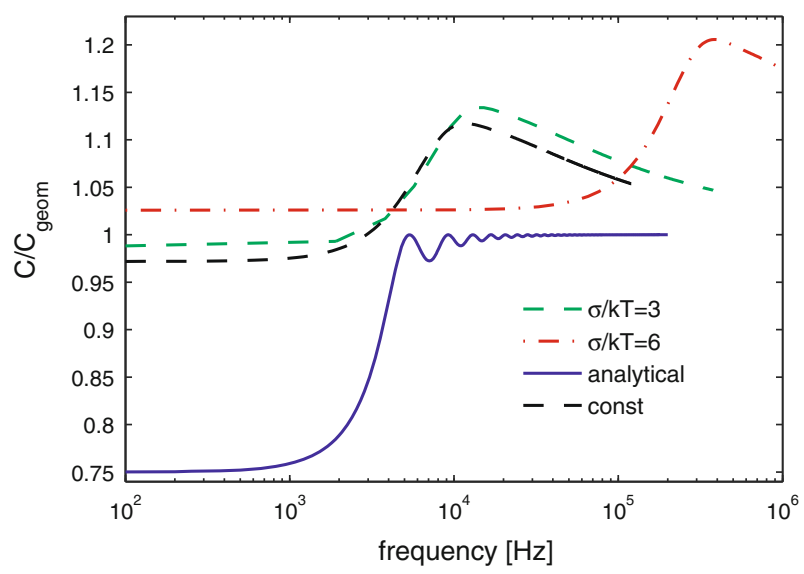

Fig. 5 Frequency dependence of the normalized capacitance at $0.5 \mathrm{~V}$ in a hole-only device for a constant mobility and diffusion coefficient, the EGDM and the analytical solution

\subsection{Impedance analysis}

As a third step, we investigate the response of the device to a voltage modulation. We obtain therefrom the impedance $Z(\omega)$ which is related to the admittance $Y(\omega)$ as $Y(\omega)=\frac{1}{Z(\omega)}$. The admittance is decomposed into an equivalent parallel conductance and capacitance $Y(\omega)=$ $G(\omega)+i \omega C(\omega)$. We analyze the normalized capacitance at $0.5 \mathrm{~V}$ in Fig. 5 and at $8 \mathrm{~V}$ in Fig. 6. In both, the low and the high voltage case, we varied the energetic disorder parameter $\sigma$ which is the main EGDM model parameter. We compare our numerical simulation results with the analytical solution for the drift-only case. For the small signal analysis the analytical solution for the impedance $Z(\omega)$ is given by Shao and Wright (1961)

$$
Z(\omega)=\frac{6}{g_{0}\left(i \omega \tau_{0}\right)^{3}}\left[1-i \omega \tau_{0}+\frac{1}{2}\left(i \omega \tau_{0}\right)^{2}-\exp \left(-i \omega \tau_{0}\right)\right]
$$

where $\tau_{0}=\frac{4}{3} \frac{L^{3}}{\mu V}$ and $g_{0}=\frac{3}{\tau_{0}} \frac{\epsilon}{L}$. We also calculated the solution for the constant mobility case. The drift-only model provides a quite accurate solution for the constant mobility case at $8 \mathrm{~V}$. At $0.5 \mathrm{~V}$ however, the drift-only model differs significantly from the constant mobility model. We observe that the oscillations at high frequencies are damped out when diffusion is present. Also due to diffusion, the capacitance at low frequencies is increased from the typical value $\frac{3}{4} C_{\text {geom }}$ of the drift-only solution, even more in case of the EGDM. In the EGDM the peak position is additionally shifted to higher frequencies.

\subsection{Trap charge dynamics}

In admittance spectroscopy an enhanced capacitance that differs heavily from Fig. 6 at low voltage can often been observed. The increased capacitance can be modeled by introducing an additional trap density of states or trap level. In the following, we look at the effect of a single trap level that is added to the HOMO. In Fig. 7 the additional hole trap level is indicated on top of the HOMO. For simplicity, we assume a constant mobility model and neglect the enhancement factors $g_{1}(p, T), g_{2}(F, T)$ and $g_{3}(p, T)$. The trap dynamics are described by the following equation 


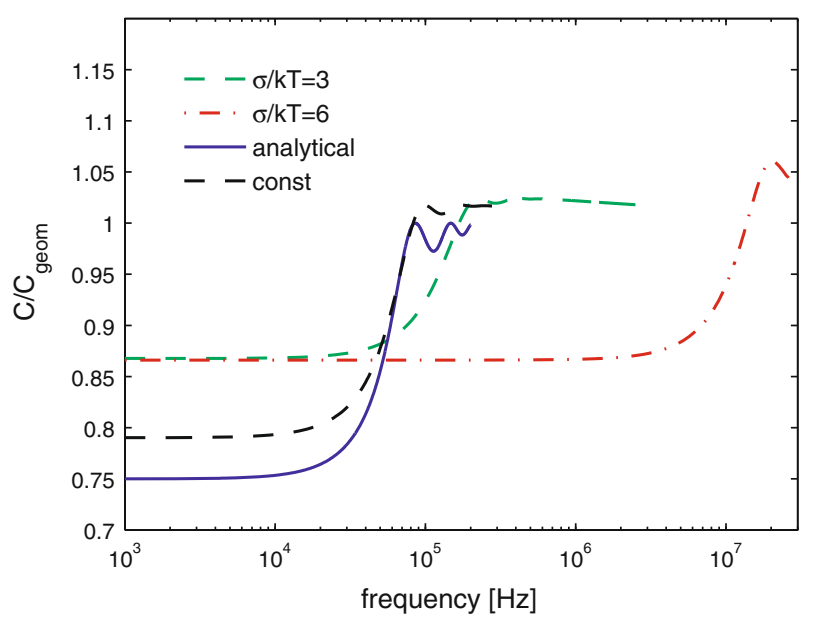

Fig. 6 Frequency dependence of the normalized capacitance at $8 \mathrm{~V}$ in a hole-only device for a constant mobility and diffusion coefficient, the EGDM and the analytical solution

Fig. 7 Illustration of a hole trap level on top of the broaden HOMO and LUMO

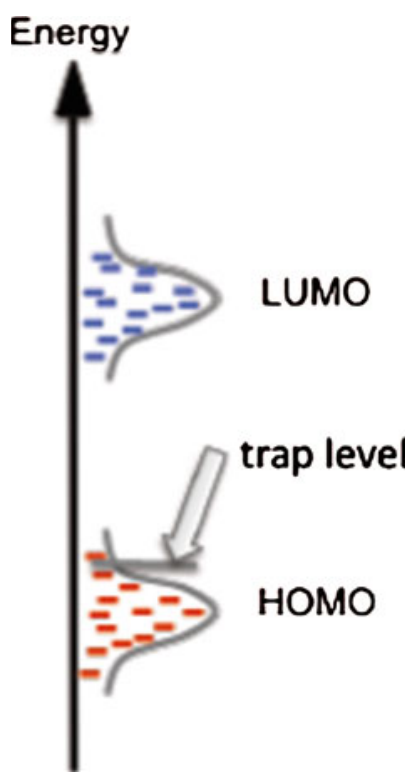

$$
\frac{\partial p_{t}}{\partial t}=c p\left(N_{t}-p_{t}\right)-e p_{t}
$$

where $c$ is the capture and $e$ the escape rate, $p_{t}$ the density of trapped holes and $N_{t}$ the total number of trap states. The trapped charge carriers also appear in Poisson's equation which is then of the following form:

$$
F_{\psi}\left(\psi, p, p_{t}\right)=\nabla \cdot(\epsilon \nabla \psi)-q\left(-p-p_{t}\right)=0,
$$

The charge continuity equation (2) is also extended by the trap dynamics (12). The smallsignal analysis is then conducted for the trap enhanced equations. Additional parameters for trap dynamics are shown in Table 2. 
Table 2 Additional simulation parameters for the inclusion of slow and fast traps, other parameters remain the same as shown in Table 1

\begin{tabular}{lll}
\hline Parameter & Value & Units \\
\hline$c_{\text {slow }}$ & $10^{-20}$ & $\mathrm{~m}^{3} / \mathrm{s}$ \\
$c_{\text {fast }}$ & $10^{-18}$ & $\mathrm{~m}^{3} / \mathrm{s}$ \\
$E_{\text {trap }}$ & $E_{\{\mathrm{HOMO}\}}+0.2$ & $\mathrm{eV}$ \\
$N_{\text {trap }}$ & $10^{24}$ & $\mathrm{~m}^{-3}$ \\
\hline
\end{tabular}

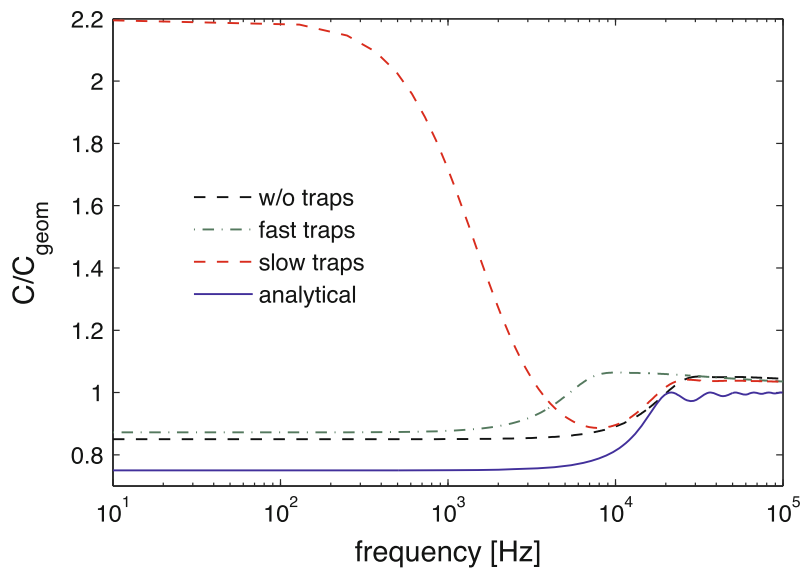

Fig. 8 Frequency dependence of the normalized capacitance in a hole-only device with fast and slow traps at $2 \mathrm{~V}$

We define fast traps in the following way: quasi-equilibrium between trapped and free carriers is maintained, the transit time is longer than the trapping time whereas for slow traps the transit time is shorter than the trapping time. In Fig. 8 we show the simulated normalized capacitance for slow and fast traps at different frequencies. Slow traps enhance the capacitance at low frequencies while fast traps follow the dynamics of the trap-free case.

For determining the mobility of charge carriers in an organic device the negative differential susceptance representation is commonly used where

$$
-\Delta B(\omega)=-\omega\left(C(\omega)-C_{\text {geom }}\right)
$$

describes the negative differential susceptance with $C_{\text {geom }}$ corresponding to the geometrical capacitance. In this representation a peak frequency $f_{\max }$ can be found which is linked to the transit time of the carriers and thus to the mobility. For the assumption of drift-only transport this leads to

$$
\mu=1.85 \frac{f_{\max } L^{2}}{\left(V-V_{b i}\right)} .
$$

It is important to see that the peak positions in Fig. 9 and therefore the apparent mobilities in this representation vary strongly if slow or fast traps are present. In the presence of fast traps there is a quasi-equilibrium between the trapped and free carriers. The trap dynamics are on a different time scale. Therefore the the peak position is shifted to lower frequencies, thus leading to a lower apparent mobility. However, for slow traps the result changes significantly at low frequency. We observe an increased capacitance at low frequency while the apparent mobility does not change a lot. 


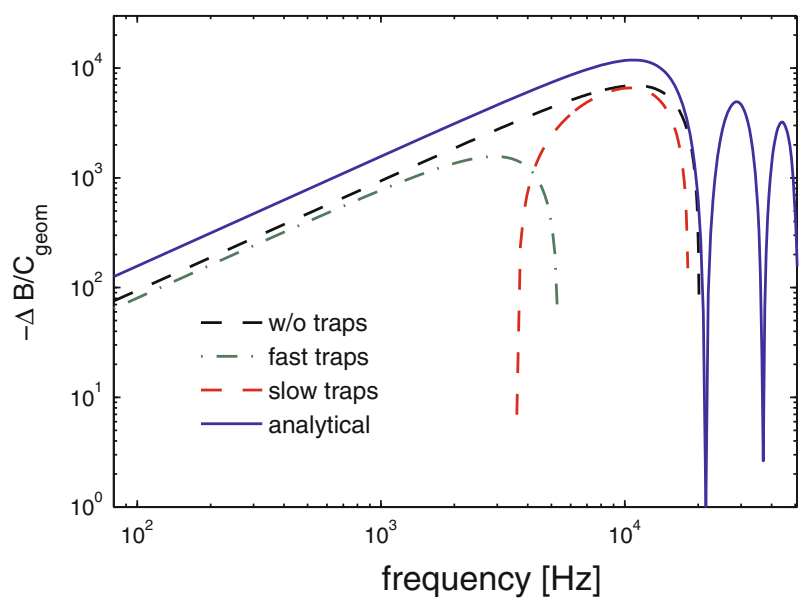

Fig. 9 Frequency dependence of the negative differential susceptance at $2 \mathrm{~V}$ for a hole-only device with fast and slow traps

\section{Conclusion and outlook}

We have investigated the influence of the EGDM, its components and the Gaussian density of states on the current-voltage curves, the dark-injection transients and the frequency response for a polymer device. All three characterization curves change significantly, if the disorder model ingredients are taken into account. Since we also show the analytical and constant mobility solutions, the deviations from simplified models are clearly visible and show the limitations of the analytical solutions obtained by the simplified drift-only model. The numerical model can be extended by a trap density of states. In this paper we have shown the influence of traps on the capacitance and the negative differential susceptance. This numerical framework provides the basis for further model validation and parameter extraction. Model parameters will be efficiently extracted from measurements with the aid of optimization algorithms. The parameters obtained for one operating condition will be checked for consistency with a different operating condition. Our numerical framework can straightforwardly be applied to more general multilayer devices that feature bipolar transport and exciton dynamics (Perucco et al. 2010).

Acknowledgments We acknowledge financial support from the European Community's Seventh Framework program under Grant Agreement No. 213708 (www.aeviom.eu).

\section{References}

Blom, P.W.M., de Jong, M.J.M., Vleggaar, J.J.M.: Electron and hole transport in poly(p-phenylene vinylene) devices. Appl. Phys. Lett. 68(23), 3308 (1996)

Crone, B.K., Davids, P.S., Campbell, I.H., Smith, D.L.: Device model investigation of bilayer organic light emitting diodes. J. Appl. Phys. 87(4), 1974 (2000)

Helfrich, W., Mark, P.: Raumladungsbeschränkte ströme in anthrazen als mittel zur bestimmung der beweglichkeit von defektelektronen. Z. Phys. 166, 370 (1962)

Knapp, E., Häusermann, R., Schwarzenbach, H., Ruhstaller, B.: Numerical simulation of charge transport in disordered organic semiconductor devices. J. Appl. Phys. 108(5), 054504 (2010)

Mott, N.P., Gurney, R.W.: Electronic Processes in Ionic Crystals. Oxford Univ. Press, London (1938) 
Pasveer, W.F., Cottaar, J., Tanase, C., Coehoorn, R., Bobbert, P.A., Blom, P.W.M., et al.: Unified description of charge-carrier mobilities in disordered semiconducting polymers. Phys. Rev. Lett. 94(20), 206601 (2005)

Perucco, B., Reinke, N.A., Müller, F., Rezzonico, D., Ruhstaller, B.: The influence of the optical environment on the shape of the emission profile and methods of its determination. Proc. SPIE 7722, 77220F (2010)

Preezant, Y., Roichman, Y., Tessler, N.: Amorphous organic devices—degenerate semiconductors. J. Phys. Cond. Matt. 14, 9913 (2002)

Roichman, Y., Tessler, N.: Generalized einstein relation for disordered semiconductors: implications for device performance. Appl. Phys. Lett. 80(11), 1948 (2002)

Ruhstaller, B., Carter, S.A., Barth, S., Riel, H., Riess, W., Scott, J.C.: Transient and steady-state behavior of space charges in multilayer organic light-emitting diodes. J. Appl. Phys. 89(8), 4575 (2001)

Ruhstaller, B., Beierlein, T., Riel, H., Karg, S., Scott, J.C., Riess, W.: Simulating electronic and optical processes in multilayer organic light-emitting devices. IEEE J. Sel. Top. Quantum Electron. 9(3), 723 (2003)

Scharfetter, D.L., Gummel, H.K.: Large-signal analysis of a silicon read diode oscillator. IEEE Trans. Electr. Dev. 16(28), 64 (1969)

Scott, J.C., Malliaras, G.G.: Charge injection and recombination at the metalorganic interface. Chem. Phys. Lett. 299, 115 (1999)

Shao, J., Wright, G.: Characteristics of the space-charge-limited dieletric diode at very high frequencies. SolidState Electron. 3, 291 (1961)

van Mensfoort, S.L.M., Coehoorn, R.: Effect of gaussian disorder on the voltage dependence of the current density in sandwich-type devices based on organic semiconductors. Phys. Rev. B 78(16), 085207 (2008)

van Mensfoort, S.L.M., Vulto, S.I.E., Janssen, R.A.J., Coehoorn, R.: Hole transport in polyfluorenebased sandwich-type devices: quantitative analysis of the role of energetic disorder. Phys. Rev. B 78(8), 085208 (2008) 